\title{
Lower Bounds on van der Waerden Numbers: Randomized- and Deterministic-Constructive
}

\author{
William Gasarch \\ Department of Computer Science \\ University of Maryland at College Park \\ College Park, MD 20742, USA \\ gasarch@cs.umd.edu
}

\author{
Bernhard Haeupler \\ CSAIL \\ Massachusetts Institute of Technology \\ Cambridge, MA 02130, USA \\ haeupler@mit.edu
}

Submitted: May 19, 2010; Accepted: Mar 8, 2011; Published: Mar 24, 2011

Mathematics Subject Classification: 05D10

\begin{abstract}
The van der Waerden number $W(k, 2)$ is the smallest integer $n$ such that every 2 -coloring of 1 to $n$ has a monochromatic arithmetic progression of length $k$. The existence of such an $n$ for any $k$ is due to van der Waerden but known upper bounds on $W(k, 2)$ are enormous. Much effort was put into developing lower bounds on $W(k, 2)$. Most of these lower bound proofs employ the probabilistic method often in combination with the Lovász Local Lemma. While these proofs show the existence of a 2-coloring that has no monochromatic arithmetic progression of length $k$ they provide no efficient algorithm to find such a coloring. These kind of proofs are often informally called nonconstructive in contrast to constructive proofs that provide an efficient algorithm.

This paper clarifies these notions and gives definitions for deterministic- and randomized-constructive proofs as different types of constructive proofs. We then survey the literature on lower bounds on $W(k, 2)$ in this light. We show how known nonconstructive lower bound proofs based on the Lovász Local Lemma can be made randomized-constructive using the recent algorithms of Moser and Tardos. We also use a derandomization of Chandrasekaran, Goyal and Haeupler to transform these proofs into deterministic-constructive proofs. We provide greatly simplified and fully self-contained proofs and descriptions for these algorithms.
\end{abstract}

\section{Introduction}

Notation 1.1 Let $[n]=\{1, \ldots, n\}$ and $\mathbf{N}^{+}=\{1,2, \ldots\}$. If $k \in \mathbf{N}^{+}$then a $k$-AP means an arithmetic progression of size $k$, i.e., $k$ numbers of the form $\{a, a+d, \ldots, a+(k-1) d\}$ with $a, d \in \mathrm{N}^{+}$.

Recall van der Waerden's theorem: 
Theorem 1.2 For every $k \geq 1$ and $c \geq 1$ there exists $W$ such that for every $c$-coloring $C O L:[W] \rightarrow[c]$ there exists a monochromatic $k$-AP, i.e. there are $a, d \in \mathrm{N}^{+}$, such that

$$
\operatorname{COL}(a)=\operatorname{COL}(a+d)=\cdots=\operatorname{COL}(a+(k-1) d) .
$$

Definition 1.3 Let $k, c, n \in \mathrm{N}$ and let $C O L:[n] \rightarrow[c]$. We say that $C O L$ is a $(k, c)$ proper coloring of $[n]$ if there is no monochromatic $k$-AP in $[n]$. We denote with $W(k, c)$ the least $W$ such that van der Waerden's theorem holds with these values of $k, c$ and $W$, i.e., the least $W$ such that there exists no proper coloring of $[W]$.

The first proof of Theorem 1.2 was due to van der Waerden [25]. The bounds on $W(k, c)$ were (to quote Graham, Rothchild, and Spencer [10]) EEEENORMOUS. Formally they were not primitive recursive. The proof is purely combinatorial. Shelah [23] gave primitive recursive bounds with a purely combinatorial proof. The best bound is due to Gowers [9] who used rather hard mathematics to obtain

$$
W(k, c) \leq 2^{2^{c 2^{2^{k+9}}}} .
$$

In this paper we survey lower bounds for van der Waerden numbers. Some of the bounds are obtained by probabilistic proofs. Since such proofs do not produce an actual coloring they are often called, informally, nonconstructive. However, since all of the objects involved are finite, one could (in principle) enumerate all of the colorings until one with the correct properties is found. We do not object to the term nonconstructive; however, we wish to clarify it. To this end we formally define two types of constructive proofs. We only define these notions for proofs of lower bounds on $W(k, c)$. It would be easy to define constructive proofs in general; however, we want to keep our presentation simple and focused.

Definition 1.4 A proof that $W(k, c) \geq f(k, c)$ is deterministic-constructive if it presents an algorithm that will, for all $k, c$, produce a proper $c$-coloring of $[f(k, c)]$ in time polynomial in $f(k, c)$.

Some of the nonconstructive techniques yield a randomized algorithm that, with high probability, will produce a proper coloring in polynomial time. These seem to us to be different from truly nonconstructive techniques. Hence we define a notion of randomizedconstructive.

Definition 1.5 A proof that $W(k, c) \geq f(k, c)$ is randomized-constructive if it presents a randomized algorithm that will, for all $k, c$,

- always produce either a proper $c$-coloring or the statement I HAVE FAILED!,

- with probability $\geq 2 / 3$ produce a proper $c$-coloring, and

- terminate in time polynomial in $f(k, c)$. 


\section{Note 1.6}

1. The success probability can be increased through standard amplification by repeating the algorithm (say) $f(k, c)$ times to make the probability of success $1-\frac{1}{3^{f(k, c)}}$ or even higher. The required explicitly declared one-sided error makes it furthermore possible to transform each randomized-constructive proof into a Las Vegas algorithm that always outputs a proper $c$-coloring in expected polynomial time.

2. Similar probabilistic proofs of lower bounds for (off-diagonal) Ramsey Numbers [10, 11] are neither deterministic-constructive nor randomized-constructive. The reason for this is that no polynomial time algorithm for detecting a failure (i.e., finding a large clique or independent set) is known. This makes randomized algorithms such as the ones by Haeupler, Saha, and Srinivasan [11] inherently Monte Carlo algorithms that cannot be made randomized-constructive.

3. Work of Wigderson et al. [13, 19] on derandomization shows that, under widely believed but elusive to prove hardness assumptions, randomness does not help algorithmically - or more formally that $\mathrm{P}=\mathrm{BPP}$. In this case the above two notions of randomized-constructive and deterministic-constructive would coincide.

We present the following lower bounds:

1. $W(k, 2) \geq \sqrt{\frac{k}{3}} 2^{(k-1) / 2}$ by a randomized-constructive proof. This is an easy and known application of the probabilistic method of Erdös and Rado [6]. This result is usually presented as being nonconstructive.

2. $W(k, 2) \geq \sqrt{k} 2^{(k-1) / 2}$ by a deterministic-constructive proof. This is an easy derandomization of the Erdös-Rado lower bound using the method of conditional expectations of Erdös and Selfridge [7]. It is likely known though we have never seen it stated.

3. If $p$ is prime then $W(p+1,2) \geq p\left(2^{p}-1\right)$ by a deterministic-constructive proof. Berlekamp [3] proved this; however, our presentation follows that of Graham et al [10]. Berlekamp actually proved $W(p+1,2) \geq p 2^{p}$. He also has lower bounds if $k$ is a prime power and $c$ is any number. Using a hard result from number theory [1] we obtain as a corollary that, for all but a finite number of $k, W(k, 2) \geq$ $\left(k-k^{0.525}\right)\left(2^{k-k^{0.525}}-1\right)$.

4. $W(k, 2) \geq \frac{2^{(k-1)}}{4 k}$ by a randomized-constructive proof. The nonconstructive version of this bound is implied by the Lovász Local Lemma [5] and by Szabó's result [24] (explained below). The randomized-constructive proof is an application of Moser's [17] algorithmic proof of the Lovász Local Lemma. Our presentation is based on Moser's STOC presentation [16] in which he sketched a Kolmogorov complexity based proof that differed significantly from the conference paper [17]. Later Moser and Tardos wrote a sequel making the general Lovász Local Lemma (with the optimal constants) 
constructive [18]. Schweitzer had, independently, used Kolmogorov complexity to obtain lower bounds on $W(k, c)[22]$.

5. For all $\epsilon>0$, for all $k \in \mathbf{N}^{+}, W(k, 2) \geq \frac{2^{(k-1)(1-\epsilon)}}{e k}$ by a deterministic-constructive proof. More precisely we give a deterministic algorithm that, given $k$ and $\epsilon$, always outputs a proper coloring of $\left[\frac{2^{(k-1)(1-\epsilon)}}{e k}\right]$ in time $2^{O(k / \epsilon)}$ which is polynomial in the output size for any constant $\epsilon>0$. This result is an application of a derandomization of the Moser-Tardos algorithm for the Lovász Local Lemma given by Chandrasekaran, Goyal and B. Haeupler [4]. We present a simplified, short and completely self-contained proof.

6. The Lovász Local Lemma algorithm by Moser and Tardos [18] can be used to obtain $W(k, 2) \geq \frac{2^{(k-1)}}{e k}$ by a randomized-constructive proof matching the best nonconstructive bound directly achievable via the Lovász Local Lemma (see [10]). We show $W(k, 2) \geq \frac{2^{(k-1)}}{e k}-1$ as a simple corollary of our deterministic-constructive proof.

\section{Note 1.7}

1. The best known (asymptotic) lower bound on $W(k, 2)$ is due to Szabó [24]:

$$
\forall \epsilon>0, \forall \text { large } k: W(k, 2) \geq \frac{2^{k}}{k^{\epsilon}} .
$$

The proof is involved, relies on the Lovász Local Lemma and additionally exploits the structure of $k$-APs that almost all $k$-AP are almost disjoint (i.e., intersect in at most one number). While the original proof is nonconstructive it can be made constructive using the methods of some recent papers $[4,11,18]$.

2. There is no analog of Szabó's bound for $c \geq 3$ colors known. In contrast to this the techniques presented here directly extend to give lower bounds on multi-color van der Waerden numbers of the form $W(k, c) \geq \frac{c^{(k-1)}}{e k}$ for any integer $c \geq 2$.

3. The techniques used to prove the results mentioned in items $1,2,3,5$, and 6 can be modified to get lower bounds for variants of van der Waerden numbers such as Gallai-Witt numbers (multi-dimensional van der Warden Numbers) [20, 21] (see also $[8,10]$ ), and some polynomial van der Waerden numbers $[2,26]$ (see also [8]).

We use the following easy lemmas throughout the paper.

Lemma 1.8 Let $k, n \in \mathrm{N}^{+}$.

1. Given a $k$-AP of $[n]$ the number of $k$-AP's that intersect it is less than $k n$.

2. The number of $k$-AP's of $[n]$ is less than $n^{2} / k$. 


\section{Proof:}

1.) We first bound how many $k$-AP's contain a fixed number $x \in[n]$. Let $1 \leq i \leq k$. If $x$ is the $i^{\text {th }}$ element of some $k$-AP then in order for this $k$-AP to be contained in $[n]$ its step width $d$ has to obey: $1 \leq x-(i-1) d$ and $x+(k-i) d \leq n$.

We assume for simplicity that $k$ is even (the odd case is nearly identical). Once $i$ and $d$ are fixed, the $k$-AP is determined. We sum over all possibilities of $i$ while assuming the second bound on $d$ for all $i \leq k / 2$ and the first bound for $i>k / 2$. This gives us the following upper bound on the number of $k$-APs going through a fixed $x$ :

$$
\begin{gathered}
\sum_{i=1}^{k / 2} \frac{n-x}{k-i}+\sum_{i=k / 2+1}^{k} \frac{x-1}{i-1}=(n-x) \sum_{i=1}^{k / 2} \frac{1}{k-i}+(x-1) \sum_{i=k / 2+1}^{k} \frac{1}{i-1}= \\
=(n-x+x-1) \sum_{i=k / 2+1}^{k} \frac{1}{i-1} \leq n-1 .
\end{gathered}
$$

Here the last inequality follows from $\sum_{i=k / 2}^{k} \frac{1}{i-1} \leq 1$ which can be easily shown by induction. Using this upper bound we get that the number of $k$-AP's that intersect a given $k$-AP is at most $k(n-1)<k n$.

2.) If a $k$-AP has starting point $a$ then then $a+(k-1) d \leq n$, so $d \leq \frac{n-a}{k-1}$. Hence, for any $a \in[n]$, there are at most $\frac{n-a}{k-1} k$-AP's that start with $a$. The total number of $k$-AP's in $[n]$ is thus bounded by

$$
\sum_{a=1}^{n-1} \frac{n-a}{k-1}=\frac{1}{k-1} \sum_{a=1}^{n-1} n-a=\frac{n(n-1)}{2(k-1)}<\frac{n^{2}}{k}
$$

\section{A Simple Randomized-Constructive Lower Bound}

Theorem 2.1 $W(k, 2) \geq \sqrt{\frac{k}{3}} 2^{(k-1) / 2}$ by a randomized-constructive proof.

Proof: We first present the classic nonconstructive proof and then show how to make it into a randomized-constructive proof.

Let $n=\sqrt{\frac{k}{3}} k 2^{(k-1) / 2}$. Color each number $x$ from 1 to $n$ by flipping a fair coin. If the coin is heads then color $x$ with 0 , if the coin is tails then color $x$ with 1 . Let $p$ be the probability that there is a monochromatic $k$-AP. We will show that $p<1$ and hence there is some choice of coin flips that leads to a proper 2-coloring of $[n]$.

By Lemma 1.8 the number of $k$-AP's is bounded by $n^{2} / k$. Because of the random choice of colors each $k$-AP becomes monochromatic with probability exactly $2^{-(k-1)}$ and a simple union bound over all $k$-AP's gives: 


$$
p \leq\left(n^{2} / k\right) 2^{-(k-1)}=\frac{n^{2}}{k 2^{(k-1)}} .
$$

Looking ahead to making this proof randomized-constructive we want this probability to be at most $1 / 3$. We show that this is implied by our choice of $n$.

$$
\begin{gathered}
\frac{n^{2}}{k 2^{k-1}} \leq 1 / 3 \\
3 n^{2} \leq k 2^{k-1} \\
\sqrt{3} n \leq \sqrt{k} 2^{(k-1) / 2} \\
n \leq \sqrt{\frac{k}{3}} 2^{(k-1) / 2} .
\end{gathered}
$$

We now present a randomized algorithm that produces (with high probability) a proper coloring and admits its failure when it does not.

1. Get input $k$ and let $n=\sqrt{\frac{k}{3}} 2^{(k-1) / 2}$.

2. Use $n$ random bits to color $[n]$.

3. Check all $k$-APs of $[n]$ to see if any are monochromatic. (by Lemma 1.8 there are at most $n^{2} / k$ different $k$-APs to check, so this takes $O\left(n^{2}\right)$ time). If none are monochromatic then the coloring is proper and we output it. Else output I HAVE FAILED!.

By the above calculations the probability of success is $\geq 2 / 3$. By comments made in the algorithm it runs in polynomial time.

\section{A Simple Deterministic-Constructive Proof}

Theorem 3.1 $W(k, 2) \geq \sqrt{k} 2^{(k-1) / 2}$ by a deterministic-constructive proof.

Proof: We derandomize the algorithm from Section 2 using the method of conditional probabilities [5]. Let $n<\sqrt{k} 2^{(k-1) / 2}$ and $X$ be the set of all arithmetic progressions of length $k$ that are contained in $[n]$.

Let $f: \mathrm{R}^{n} \rightarrow \mathrm{R}$ be defined by

$$
f\left(x_{1}, \ldots, x_{n}\right)=\sum_{s \in X}\left(\prod_{i \in s} x_{i}+\prod_{i \in s}\left(1-x_{i}\right)\right) .
$$


We will color $[n]$ with 0 's and 1's. Assume we have such a coloring and that $x_{i}$ is the color of $i$. When $x_{i}$ is set to $1 / 2$ that means that we have not colored it yet. Note that $f\left(x_{1}, \ldots, x_{n}\right)$ gives exactly the expected number of monochromatic $k$-AP's when each number $i$ gets colored independently with probability $P(i$ is colored 1$)=x_{i}$. Thus a coloring has a monochromatic $k$-AP iff $f\left(x_{1}, \ldots, x_{n}\right) \geq 1$. We will color $[n]$ such that $f\left(x_{1}, \ldots, x_{n}\right)<1$.

Note that

$$
\begin{aligned}
f(1 / 2, \ldots, 1 / 2)=\sum_{s \in X}\left(\prod_{i \in s} 1 / 2+\prod_{i \in s} 1 / 2\right) & =\sum_{s \in X}\left((1 / 2)^{k}+(1 / 2)^{k}\right) \\
& =\sum_{s \in X}(1 / 2)^{k-1} \\
& \leq n^{2} /\left(k 2^{k-1}\right)
\end{aligned}
$$

We need this to be $<1$. We set this $<1$ which will derive what $n$ has to be.

$$
\begin{aligned}
n^{2} /\left(k 2^{k-1}\right) & <1 \\
n^{2} & <k 2^{k-1} \\
n & <\sqrt{k} 2^{(k-1) / 2}
\end{aligned}
$$

We now present a deterministic algorithm:

1. Let $x_{1}=x_{2}=\cdots=x_{n}=1 / 2$. By Lemma 1.8 the number of $k$-AP's is $\leq n^{2} / k$. By the above calculation $f\left(x_{1}, \ldots, x_{n}\right)<1$.

2. For $i=1$ to $n$ do the following. When we color $i$ we already have $1,2, \ldots, i-1$ colored. Let the colors be $c_{1}, \ldots, c_{i-1}$. Hence our function now looks like, leaving the color of $i$ a variable, $f\left(c_{1}, \ldots, c_{i-1}, z, 1 / 2, \ldots, 1 / 2\right)$. This is a linear function of $z$. We know inductively that if $z=1 / 2$ then the value is $<1$. If the coefficient of $z$ is positive then color $i$. If the coefficient of $z$ is negative then color $i 1$. In either case this will ensure that

$$
f\left(c_{1}, \ldots, c_{i}, 1 / 2, \ldots, 1 / 2\right) \leq f\left(c_{1}, \ldots, c_{i-1}, 1 / 2, \ldots, 1 / 2\right)<1 .
$$

At the end we have $f\left(x_{1}, \ldots, x_{n}\right)<1$ and hence we have a proper 2-coloring. It is easy to see that this algorithms runs in time polynomial in $n$.

\section{An Algebraic Lower Bound}

We will need the following facts.

Fact 4.1 Let $p \in \mathbf{N}$ (not necessarily a prime).

1. There is a unique (up to isomorphism) finite field of size $2^{p}$. We denote this field by $F_{2^{p}} . F_{2^{p}}$ can be represented by $F_{2}[x] /<i(x)>$ where $i$ is an irreducible polynomial of degree $p$ in $F_{2}[x] . F_{2^{p}}$ can be viewed as a vector space of dimension $p$ over $F_{2}$. The basis of this vectors space is (the equivalence classes of) $1, x, x^{2}, \ldots, x^{p-1}$. 
2. The group $F_{2^{p}}-\{0\}$ under multiplication is isomorphic to the cyclic group on $2^{p}-1$ elements. Hence it has a generator $g$ such that

$$
F_{2^{p}}-\{0\}=\left\{g, g^{2}, g^{3}, \ldots, g^{2^{p}-1}\right\} .
$$

This generator can be found in time polynomial in $2^{p}$.

3. Assume $p$ is prime. Let $g$ be a generator of $F_{2^{p}}$, and $\beta=g^{d}$ where $1 \leq d<2^{p}-1$. We do all arithmetic in $F_{2^{p}}$. Let $P$ be a nonzero polynomial of degree $\leq p-1$, with coefficients in $\left\{0,1,2, \ldots, 2^{p}-1\right\}$. Then $P(g) \neq 0$ and $P(\beta) \neq 0$.

Proof: The first two facts are well known and hence we omit the proof. To see the third fact note that $F_{2^{p}}$ can be viewed as a vector space of dimension $p$ over $F_{2}$. There can be no field strictly between $F_{2}$ and $F_{2^{p}}$ : if there was then its dimension as a vector space over $F_{2}$ would be a proper divisor of $p$. For any $a \in F_{2^{p}}-F_{2}$ we get now that $F_{2}(a)$ is $F_{2^{p}}$ because it would otherwise be a field strictly between $F_{2}$ and $F_{2^{p}}$. Hence the minimal polynomial of $a$ in $F_{2}[X]$, which we denote $Q$, has degree $p$. Let $P$ be a nonzero polynomial in $F_{2}[X]$ of degree at most $p-1$. If $P(a)=0$ then $P$ has to be a multiple of $Q$. Since $P$ has degree $\leq p-1$ and $Q$ has degree $p$, this is impossible. Hence $P(a) \neq 0$. This applies to $a=g$ and to $a=g^{d}$ with $1 \leq d \leq 2^{p}-2$. (Note that $d=2^{p}-1$ gives $\beta=1$.)

Theorem 4.2 If $p$ is prime then $W(p+1,2) \geq p\left(2^{p}-1\right)$ by a deterministic-constructive proof.

Proof: Let $F=F_{2^{p}}$, the field on $2^{p}$ elements. By Fact $1 F$ is a vector space of dimension $p$ over $F_{2}$. Let $v_{1}, \ldots, v_{p}$ be a basis. By Fact 2 there exists a generator $g$ such that

$$
F-\{0\}=\left\{g, g^{2}, g^{3}, \ldots, g^{2^{p}-1}\right\} .
$$

We express $g, g^{2}, \ldots, g^{2^{p}-1}, g^{2^{p}}, \ldots, g^{p\left(2^{p}-1\right)}$ in terms of the basis. This looks odd since $g=g^{2^{p}}$ so this list repeats itself; however, it will be useful.

For $1 \leq j \leq p\left(2^{p}-1\right)$ and for $1 \leq i \leq p$ let $a_{i j} \in\{0,1\}$ be such that

$$
g^{j}=\sum_{i=1}^{p} a_{i j} v_{i}
$$

We now color $\left[p\left(2^{p}-1\right)\right]$. Let $j \in\left[p\left(2^{p}-1\right)\right]$. Color $j$ with $a_{1 j}$. That is, express $g^{j}$ in the basis $\left\{v_{1}, \ldots, v_{p}\right\}$ and color it with the coefficient of $v_{1}$, which will be a 0 or 1 . We need to show that this is indeed a proper coloring. Assume, by way of contradiction that the coloring is not proper. Hence there is a monochromatic $(p+1)$-AP. We denote it

$$
a, a+d, \ldots, a+p d .
$$

Since all of the numbers are in $\left[p\left(2^{p}-1\right)\right]$ we have $a+p d \leq p\left(2^{p}-1\right)$ and thus $d \leq 2^{p}-2$. Therefore we get $g^{d} \neq 1$. 
If we express any of

$$
I=\left\{g^{a}, g^{a+d}, \ldots, g^{a+p d}\right\}=\left\{g^{a}, g^{a} g^{d}, g^{a} g^{2 d}, \ldots, g^{a} g^{p d}\right\}
$$

in terms of the basis they have the same coefficient for $v_{1}$. Let $\alpha=g^{a}$ and $\beta=g^{d} \neq 1$. Recall that, by Fact $3, \beta$ does not solve any degree $p-1$ polynomial with coefficients in $\{0,1\}$.

Case 1: The coefficient is 0 . Then we have that all of the elements of $I$ lie in the $p-1$ dim space spanned by $\left\{v_{2}, \ldots, v_{p}\right\}$. There are $p+1$ elements of $I$, so any $p$ of them are linearly dependent. Hence $I^{\prime}=\left\{\alpha, \alpha \beta, \alpha \beta^{2}, \ldots, \alpha \beta^{p-1}\right\}$ is linearly dependent. So there exists $b_{0}, \ldots, b_{p-1} \in\{0,1\}$, not all 0 , such that

$$
\begin{gathered}
\sum_{i=0}^{p-1} b_{i} \alpha \beta^{i}=0 \\
\sum_{i=0}^{p-1} b_{i} \beta^{i}=0 .
\end{gathered}
$$

Therefore $\beta$ satisfies a polynomial of degree $\leq p-1$ with coefficients in $\{0,1\}$, contradicting Fact 3.

Case 2: The coefficient is 1 . Hence all of the elements of $I$, when expressed in the basis $\left\{v_{1}, \ldots, v_{p}\right\}$ have coefficient 1 for $v_{1}$. Take all of the elements of $I$ (except $\alpha$ ) and subtract $\alpha$ from them. The set we obtain is

$$
\left\{\alpha \beta-\alpha, \alpha \beta^{2}-\alpha, \ldots, \alpha \beta^{p}-\alpha\right\}=\left\{\alpha(\beta-1), \alpha\left(\beta^{2}-1\right), \ldots, \alpha\left(\beta^{p}-1\right)\right\} .
$$

KEY: All of these elements, when expressed in the basis, have coefficient 0 for $v_{1}$. Hence we have $p$ elements in a $p-1$-dim vectors space. Therefore they are linearly dependent. So there exists $b_{0}, \ldots, b_{p-1} \in\{0,1\}$, not all 0 , such that

$$
\begin{aligned}
& \sum_{i=0}^{p-1} b_{i} \alpha\left(\beta^{i}-1\right)=0 \\
& \sum_{i=0}^{p-1} b_{i}\left(\beta^{i}-1\right)=0 .
\end{aligned}
$$

Therefore $\beta$ satisfies a polynomial of degree $\leq p-1$ over $F_{2}$. This contradicts Fact 3 . We now express the above proof in terms of a deterministic construction.

1. $\operatorname{Input}(p+1)$.

2. Find an irreducible polynomial $i(x)$ of degree $p$ over $F_{2}[x]$. This gives a representation of $F_{2^{p}}$, namely $F_{2}[x] /<i(x)>$. Note that $1, x, x^{2}, \ldots, x^{p-1}$ is a basis for $F_{2^{p}}$ over $F_{2}$. Let $v_{i}=x^{i+1}$. 
3. Find $g$, a generator for $F_{2^{p}}$ viewed as a cyclic group.

4. Express $g, g^{2}, \ldots, g^{p\left(2^{p}-1\right)}$ in terms of the basis. For $1 \leq j \leq p\left(2^{p}-1\right)$, for $1 \leq i \leq p$ let $a_{i j} \in\{0,1\}$ be such that $g^{j}=\sum_{i=1}^{p} a_{i j} v_{i}$.

5. Let $j \in\left[p\left(2^{p}-1\right)\right]$. Color $j$ with $a_{1 j}$.

Steps 2 and 3 can be done in time polynomial in $2^{p}$ by Fact 4.1 . Step 4 can be done in time polynomial in $2^{p}$ using simple linear algebra. Hence the entire algorithm takes time polynomial in $2^{p}$.

Baker, Harman, and Pintz [1] (see [12] for a survey) showed that, for all but a finite number of $k$, there is a prime between $k$ and $k-k^{0.525}$. Hence we have the following corollary.

Corollary 4.3 For all but a finite number of $k$,

$$
W(k, 2) \geq\left(k-k^{0.525}\right)\left(2^{k-k^{0.525}}-1\right) .
$$

(We do not claim this proof is deterministic-constructive or randomized-constructive.)

Proof: Given $k$ let $p$ be the primes such that $k-k^{0.525} \leq p \leq k$. By Theorem 4.2 $W(p+1,2) \geq p\left(2^{p}-1\right)$ Hence

$$
W(k, 2) \geq W(p+1,2) \geq p\left(2^{p}-1\right) \geq\left(k-k^{0.525}\right)\left(2^{k-k^{0.525}}-1\right) .
$$

\section{A Bit of Kolmogorov Theory}

We will need some Kolmogorov theory for the next section and thus give a short introduction here. For a fuller and more rigorous account of Kolmogorov Theory see the book by Li and Vitanyi [15].

What makes a string random? Consider the string $x=0^{n}$. This string does not seem that random but how can we pin that down? Note that $x$ is of length $n$ but can be easily produced by a program of length $\lg (n)+O(1)$ like this:

FOR $x=1$ to $n, \operatorname{PRINT}(0)$

By contrast consider the following string

$$
x=0110100101010010101011111100001110010101
$$

which we obtained by flipping a coin 40 times. It can be produced by the following program.

PRINT(0110100101010010101011111100001110010101) 
Note that this program is of length roughly $|x|$. There does not seem to be a shorter program to produce $x$. The string $x$ seems random in that there is no pattern in $x$ which would lead to a shorter program to print $x$ than the one above. Informally a string $x$ looks random, if the shortest program to print out $x$ has length roughly $|x|$. We formalize this.

Definition 5.1 Fix a programming language $L$ that is Turing complete. Let $x \in\{0,1\}^{n}$ (think of $n$ as large) and $y \in\{0,1\}^{m}$ (think of $m$ as small). $K_{L}(x \mid y)$ is the length of the shortest program $P$ in $L$ such that $P(y)$ has output $x$.

Fact 5.2 If $L_{1}$ and $L_{2}$ are Turing complete programming languages then there is a program that translates one to the other. This program is of constant size. Hence there is a constant $\alpha \in \mathrm{N}$ such that $\left|K_{L_{1}}(x \mid y)-K_{L_{2}}(x \mid y)\right| \leq \alpha$. Therefore $K_{L}(x \mid y)$ is independent of $L$ up to an additive constant factor. Hence we will drop the $L$ and always include an $O(1)$ or $\Omega(1)$ term as is appropriate.

Definition 5.3 A string $x$ is Kolmogorov random relative to $y$ if $K(x \mid y) \geq|x|+\Omega(1)$.

Fact 5.4 By comparing the number of strings of length $n$ to the number of descriptions of length smaller than $n$ we conclude that most strings are Kolmogorov random. Hence if you find that a randomized algorithm works well when you use a Kolmogorov random string for the random bits, then it works well for most strings. We will assume that at least $2 / 3$ of all strings of length $n$ are Kolmogorov random; however, there are really far more.

\section{A Randomized-Constructive Lower Bound via the Lovász Local Lemma}

We use the following lemma both in this section and the next section. The bulk of this lemma is an exercise from Knuth [14]; however, we include the proof for completeness.

Lemma 6.1 Let $m \in \mathrm{N}$ and $T, T_{1}, \ldots, T_{m}$ be infinite rooted trees with each node having exactly $x$ ordered children.

1. There are at most $\left(\begin{array}{c}x s \\ s\end{array}\right) \leq(e x)^{s}$ subtrees of $T$ that include the root and consist of exactly s non-root nodes.

2. Let $\mathcal{F}$ be the set of all forests $F$ consisting of at most $m$ trees, such that each tree is a subtree of a different $T_{i}$, and such that the total number of nodes in $F$ is $s . \mathcal{F}$ consists of at most $2^{m}(e x)^{s}$ forests. 


\section{Proof:}

1.) Given a subtree of $T$ with $s$ non-root nodes, record an ordered DFS traversal using a zero to denote that a potential child is not there and a one for every forward step along an existing child. Stop the traversal at the last non-root node without recording zeros for its children. There are $x$ (potential) children each for both the root and each but the last of the $s$ non-root nodes; each of these $s x$ children appears at most once in the traversal. Therefore a string of length at most $s x$ is recorded. The string has furthermore exactly $s$ ones, one for each non-root node. Note that any two different subtrees of $T$ correspond to two different strings. We thus have an injection from the specified subtrees into the set of zero-one strings of length at most $x s$ with exactly $s$ ones. There are exactly $\left(\begin{array}{c}s x \\ s\end{array}\right)$ such strings and therefore also at most this many subtrees of $T$ with $s$ non-root nodes. The inequality $\left(\begin{array}{c}s x \\ s\end{array}\right)<(x e)^{s}$ follows from Stirling's formula.

2.) Let $T^{\prime}$ be the ordered tree that has a root $r$ of degree $m$ and at the $i^{\text {th }}$ child of $r$ attached $T_{i}$ (so the $i^{t h}$ node on the second level is the root of $T_{i}$ ). There is a straight forward bijection between forests in $\mathcal{F}$ and subtrees of $T^{\prime}$ with $s$ non-root nodes.

We describe such a subtree of $T^{\prime}$ by a subset of $[m]$ to specify the children of $r$ that are not used and by a zero-one string of length at most $x s$ with exactly $s$ ones corresponding to a DFS-traversal of the remaining tree in the same manner as above. Each forest in $\mathcal{F}$ can be uniquely described in such a manner (but not all those descriptions correspond to a valid tree). The total number of those descriptions and therefore also the total number of forests in $\mathcal{F}$ is at most $2^{m}(x e)^{s}$.

Theorem $6.2 W(k, 2) \geq \frac{2^{(k-1)}}{4 k}$ by a randomized-constructive proof.

Proof: Let $n=\frac{2^{(k-1)}}{4 k}$. We present a randomized algorithm to find a 2-coloring of $[n]$. Let $E_{1}, \ldots, E_{m}$ be the $k$-AP's of $[n]$ listed in lexicographic order. By Lemma 1.8, $m=O\left(n^{2} / k\right)$.

We present a simple algorithm with a parameter $s$, which we will determine later.

\section{MAIN ALGORITHM}

1. Color $[n]$ using $n$ random bits

2. $N U M C A L L S=0$ (this will be the number of calls to FIX).

3. For $i=1$ to $m$ if $E_{i}$ is monochromatic then $\operatorname{FIX}\left(E_{i}\right)$.

4. Output the coloring.

\section{END OF MAIN ALGORITHM}

FIX ALGORITHM

$F I X(E)$

1. $N U M C A L L S=N U M C A L L S+1$. 
2. If $N U M C A L L S=s$ then STOP and output I HAVE FAILED.

3. Recolor $E$ randomly (this takes $k$ random bits).

4. While there exists a monochromatic $k$-AP that intersects $E$ let $E^{\prime}$ be the lexicographic smallest such $k$-AP and call $F I X\left(E^{\prime}\right)$.

\section{END OF FIX ALGORITHM}

We leave the following easy claims to the reader:

Claim 1: For all calls to FIX that terminate the following holds: all of the $k$-AP's that were not monochromatic before the call are not monochromatic after the call.

Claim 2: If the algorithm outputs a coloring then it is a proper coloring.

We find a value for the parameter $s$ such that $s$ is polynomial in $n$ and $k$ and such that the probability of the algorithm's success is at least $2 / 3$. With parameter $s$ the algorithm uses at most $n+s k$ random bits. We can think of the algorithm as a deterministic one which takes an additional $n+s k$ bit string as input to use in place of the random bits. Let $z=z_{0} z_{1} \cdots z_{s}$ denote that string. The first $n$ bits are used for the initial color assignment, and the remaining bits are used for the reassignments as needed.

Let $z=z_{0} z_{1} \cdots z_{s}$ be a Kolmogorov random string relative to $k, n$. We will show that if the algorithm is run with $z$ supplying the random bits then the result will be a proper coloring of $[n]$. Since over $2 / 3$ of all strings of length $n+s k$ are Kolmogorov random relative to $k, n$ this will prove that the algorithm succeeds with probability $\geq 2 / 3$.

Assume, by way of contradiction, that the algorithm goes through $s$ calls to FIX. We will pick a value of $s$ so that this leads to a contradiction.

Definition 6.3 The FIX-FOREST is the forest of calls to FIX. We take the children of a node to be ordered in the same order the procedure FIX was called. The nodes are labeled by what monochromatic $k$-AP they were called with and what color (a bit) the $k$-AP was before the call.

Definition 6.4 For $1 \leq i \leq m$ we define a tree $T_{i}$ as follows.

- The root is labeled with $E_{i}$ (the $i^{t h} k$-AP in lexicographic order).

- If a node is labeled with a $k$-AP $E$ then the children are the $k$-AP's that intersect $E$ in lexicographic order.

Putting all this together we get:

\section{Fact 6.5}

1. By Lemma 1.8 every node of $T_{i}$ has at most $k n$ children.

2. By Claim 1 the FIX-FOREST has less than $n^{2} / k$ trees 


\section{All trees in the forest are subtrees of different subtrees $T_{i}$.}

This makes it possible to apply Lemma 6.1 and obtain that the number of different FIX-FOREST structures is at most $2^{\frac{n^{2}}{k}}(k n)^{s}$. From this we get that, given $n$ and $k$, each FIX-FOREST can be described by $\frac{n^{2}}{k}+s \lg (k n)+O(1)$ bits for its structure and another $s$ bits for the color labels. Now let $w$ be the coloring after $s$ calls to FIX are performed. Note that $w$ can be described with $n$ bits. The next claim shows that taking all these descriptions it is possible to reconstruct the Kolmogorov random string $z$.

Claim 3: Given $n, k$ the FIX FOREST and $w$ one can recover $z$. Proof of Claim 3

From the FIX FOREST we can obtain:

- a description of the $k$-AP $a_{i}$ that the $i^{t h}$ call was made on.

- the color $c_{i}$ of $a_{i}$ when the $i^{\text {th }}$ call to FIX was made.

We recover the $z$ 's in three phases.

Phase I (just use the $a_{i}$ 's but not the $c_{i}$ 's): Simulate the Coloring Algorithm using the symbols $z_{i}^{j}$ where $(0 \leq i \leq s$, if $i=1$ then $1 \leq j \leq n$, if $i \geq 2$ then $1 \leq j \leq k)$ to represent the $j$ th bit of $z_{i}$. Note that we do not know the actual colors so we really do use (say) $z_{3}^{4}$ and not RED (or more formally 0 or 1 ). Since we have $a_{i}$ we can (and do) keep track of the coloring of $[n]$ after each call to FIX, in terms of the symbols $z_{i}^{j}$. This creates a table of $z_{i}^{j}$ 's.

For example, if $n=15$ then the first row will be:

$$
1 \| z_{0}^{1}\left|z_{0}^{2}\right| z_{0}^{3}\left|z_{0}^{4}\right| z_{0}^{5}\left|z_{0}^{6}\right| z_{0}^{7}\left|z_{0}^{8}\right| z_{0}^{9}\left|z_{0}^{10}\right| z_{0}^{11}\left|z_{0}^{12}\right| z_{0}^{13}\left|z_{0}^{14}\right| z_{0}^{15} \mid
$$

If $k=4 a_{1}=(4,7,10,13)$, i.e., the first call to FIX was to $(4,7,10,13)$, then the second row will be

$$
2 \| z_{0}^{1}\left|z_{0}^{2}\right| z_{0}^{3}\left|z_{1}^{1}\right| z_{0}^{5}\left|z_{0}^{6}\right| z_{1}^{2}\left|z_{0}^{8}\right| z_{0}^{9}\left|z_{1}^{3}\right| z_{0}^{11}\left|z_{0}^{12}\right| z_{1}^{4}\left|z_{0}^{14}\right| z_{0}^{15} \mid
$$

Phase II (use the $c_{i}$ 's to determine $z_{i}^{j}$ 's): For all $1 \leq i \leq s$, right before the $i^{\text {th }}$ call to $F I X, k$-AP $a_{i}$ was monochromatic; all $k$ vertices of $a_{i}$ were colored $c_{i}$. For $1 \leq j \leq i-1$ let $V_{j}$ be the vertices of $a_{i}$ that were most recently colored by $z_{j}$ (note that $V_{j}$ could be empty). By Phase I we know which bits of $z_{j}$ colored which vertices of $V_{j}$. We now know that those bits are $c_{i}$. For each $i$ we have recovered $k$ bits of $z$. Since there are $s$ calls to FIX this phase recovers $s k$ bits.

Phase III (use $w$ ): For each $x \in[n]$ there is an $i, j$ so that $x$ was colored $z_{i}^{j}$ and never recolored. We now know the $z_{i}^{j}=w^{x}$. This phase recovers $n$ bits of $z$.

The phases all together recover $n+s k$ bits of $z$. Since $|z|=n+s k$ all of $z$ is recovered.

\section{End of Proof of Claim 3}


By Claim 3, $z$ can be described using $n, k$ the FIX FOREST and $w$. Since $w$ can be described by $n$ bits and since Fact 6.5.4 tells us that the FIX FOREST can be described with $s+\frac{n^{2}}{k}+s \lg (k n)+O(1)$ bits we get a description of $z$ of size $s+\frac{n^{2}}{k}+s \lg (k n)+O(1)+n$.

On the other hand we assumed $z$ to be Kolmogorov random relative to $k, n$ which implies that any description of $z$ has to have length at least $n+s k+O(1)$. Hence

$$
\begin{gathered}
s+\frac{n^{2}}{k}+s \lg (k n)+O(1)+n \geq n+s k \\
\frac{n^{2}}{k}+O(1) \geq s k-s-s \lg (k n) \\
s \leq \frac{\frac{n^{2}}{k}+O(1)}{k-1-\lg (k n)}<n^{2} / k^{2}+O(1)
\end{gathered}
$$

Now choosing $s \geq \frac{n^{2}}{k^{2}}+O(1)$ leads to the desired contradiction.

\section{A Deterministic-Constructive Lower Bound by Derandomizing the Lovász Local Lemma}

Theorem 7.1 Fix $\epsilon>0$. W $(k, 2) \geq \frac{2^{(k-1)(1-\epsilon)}}{e k}$ by a deterministic-constructive proof.

Proof: $\quad$ Let $n=\frac{2^{(k-1)(1-\epsilon)}}{e k}$. (We assume $n$ is an integer; the modifications to make this rigorous are easy but cumbersome.) We will present a deterministic algorithm that always produces a proper 2-coloring of $[n]$ and runs in time $n^{O\left(\epsilon^{-1.01}\right)}$ which is polynomial as long as $\epsilon$ is any fixed constant. The algorithm proceeds in stages.

Let $t$ be a parameter to be named later. It will be $O\left(\epsilon^{-1.01}\right)$.

\section{Stage 1: List out Trees}

We create by exhaustive enumeration a list of the following set of trees $Y$ :

For each $k$-AP $E$, for each subset $S$ of $E$, take all possible labeled trees that satisfy the following properties:

1. the root is labeled with $S$,

2. each non-root node is labeled with a $k$-AP of $[n]$,

3. the labels of each child of a node $B$ share a number with the label of $B$,

4. the labels of nodes on the same level are disjoint,

5. there are between $t$ and $2 t$ non-root nodes. 


\begin{tabular}{|c|c|c|}
\hline 23 & 246 & 35 \\
\hline I & I & 11 \\
\hline 234 & $\begin{array}{c}567 \\
1\end{array}$ & $123 \quad 567$ \\
\hline $\begin{array}{r}23 \\
1\end{array}$ & 135 & \\
\hline 357 & $\begin{array}{l}34 \\
1 \quad 1\end{array}$ & $\begin{array}{l}4 \\
1\end{array}$ \\
\hline 3 & $123 \quad 456$ & 147 \\
\hline I & & 1 \\
\hline 123 & 14 & 357 \\
\hline | & 11 & \\
\hline 123 & $135 \quad 246$ & \\
\hline
\end{tabular}

To bound the running time of this and the next stage we need to check that the number of trees in $Y$ is always polynomial in $n$. For this recall that by Lemma 1.8 there are at most $n^{2} / k$ different $k$-AP's which gives us at most $2^{k} n^{2} / k$ possible roots. If the root is fixed then by property 3 and again Lemma 1.8 we know that each tree in $Y$ is a subtree of the infinite tree in which each $k$-AP has as children the $<n k k$-APs it intersects with. Using Lemma 6.1.2 with $x=k n$ and $s=t$ we obtain that there are at most

$$
(n k e)^{2 t}
$$

such subtrees of size $2 t$. Therefore the total number of trees $|Y|$ is at most

$$
\frac{2^{k} n^{2}}{k}(n k e)^{2 t} t \leq n^{3} \cdot\left(n^{2}\right)^{2 t} n \leq n^{O(t)}=n^{O\left(\epsilon^{-1.01}\right)} .
$$

Hence this stage of the algorithm runs in polynomial time for any fixed constant $\epsilon>0$.

\section{Stage 2: Creation of a good table}

Similar to the proof of Theorem 6.2 we create a table with a sequence of colors for every number. A table is a map $T:[n] \times[t] \rightarrow\{0,1\}$ in which we view each row as a sequence of colors for its (column)-number. We will be looking at colorings of the numbers on the nodes of the tree that is guided by a table $T$. $T(x, t)$ will tell us how to color the number $x$ the $t^{\text {th }}$ time we look for a color of $x$ when we process the tree level-by-level from leaf-to-root. More formally we assign each number $x$ in the label of a node $v \in \tau$ the color

$$
T(x, 1+\text { number of nodes below } v \text { whose label contain } x) .
$$

Given a tree $\tau$ and a coloring of its numbers guided by table $T$ say $\tau$ is consistent with $T$ iff all labels of $\tau$ are colored monochromatically. 
Note that because of property 4 each color in the table $T$ gets used only once during this process. Thus if all colors in $T$ are chosen independently at random each label of a node gets monochromatic independently with probability $2^{-(k-1)}$. The probability for a tree in $Y$ to be consistent is thus at most $2^{-(k-1) i}$ where $i$ is the number of non-root nodes. Having this and computing $\left(\frac{n^{2}}{k} 2^{k}(n k e)^{i}\right)$ as an upper bound for the number of trees with $i$ non-root nodes as in Stage 1 we get that the expectation of the number of consistent trees $X$ is at most

$$
E[X] \leq \sum_{i=t}^{2 t}\left(n^{2} 2^{k}(n k e)^{i}\right) 2^{-(k-1) i}=n^{2} 2^{k} \sum_{i=t}^{2 t} 2^{-(k-1) \epsilon i}<n^{2} 2^{k} t 2^{-(k-1) \epsilon t} \leq 2^{O(k)} t 2^{-k \epsilon t} .
$$

Thus the expectation is $<1 / 3$ if $t=O\left(\epsilon^{-1.01}\right)$ is picked large enough. Markov's inequality proves that with probability at least $\frac{2}{3}$ no tree in $Y$ is consistent with a randomly chosen table. We efficiently construct such a table using the method of conditional expectations in the following algorithm:

\section{TABLE CREATION ALGORITHM}

- For all $x=1$ to $n$, For all $y=0$ to $2 t$

- Set $T(x, y)=1 / 2$

- For all $x=1$ to $n$, For all $y=0$ to $2 t$

- Set $T(x, y)=0$

For all $\tau \in Y$

- Compute $p_{\tau, 0}=\prod_{v \in \tau}\left(\prod_{i \in \operatorname{label}(v)} \operatorname{color}(i)+\prod_{i \in \operatorname{label}(v)}(1-\operatorname{color}(i))\right)$

- (Here color $(i)$ corresponds to the entry from $T$ that is assigned to this number $i$ in the label of node $v$ when the coloring of $\tau$ is guided by $T$. Note that $p_{\tau, 0}$ corresponds exactly to the probability that every node-label in $\tau$ becomes monochromatic if colors are filled in from the table $T$ into $\tau$ level-by-level from leaf-to-root while choosing a random color instead of any $1 / 2$.)

Let $E_{0}=\sum_{\tau \in Y} p_{\tau, 0}$

- set $T(x, y)=1$ and compute all $p_{\tau, 1}$ and $E_{1}$ similarly to the last step

- if $E_{0}<E_{1}$ than $T(x, y)=0$ else $T(x, y)=1$ in order to minimize the expectation.

\section{END OF TABLE CREATION ALGORITHM}

For the analysis of the table creation we see that $E_{0}$ and $E_{1}$ are exactly the expected number of consistent trees in $Y$ if we set $T(x, y)$ to 0 or 1 respectively. Because of our choice of $t$ from above we get that in the beginning this expectation is $E=\frac{E_{0}+E_{1}}{2}<2 / 3$. 
By always choosing the color that minimizes this expectation the invariant $\frac{E_{0}+E_{1}}{2}<2 / 3$ is preserved throughout the algorithm. When finally all entries of $T$ are chosen, no randomness remains and the invariant implies that no tree with properties 1-5 is consistent with $T$. This stage of the algorithm takes $O(4 t k|Y|)$ time for each of the $2 t n$ iterations and therefore runs in time polynomial in $n$.

\section{Stage 3: Run a Recoloring Algorithm using Colors from the Table}

1. Initially color $[n]$ using the first column of $T$.

2. WHILE there is a monochromatic $k$-AP $E$ recolor the numbers in $E$ using for each number its next unused color from $T$

This completes the algorithm. In the rest of this section we show that the algorithm terminates without requesting more than $t$ colors for one number which will be enough to argue a quick termination. Note that because of the termination condition of the algorithm no proof of correctness is needed.

Claim: Each number gets recolored at most $t$ times.

\section{Proof of Claim}

Lets look at the sequence of $k$-APs as picked by the algorithm. For each $k$-AP $E$ in this sequence and each subset $S$ of $E$ we construct a tree labeled by subsets of $[n]$ by starting with a root with label $S$. Going back in the sequence we iteratively take the next $k$-AP $B$ and if there is a node in the tree whose label shares a number with $B$ we create a new node with label $B$ and attach it to the lowest such node breaking ties arbitrarily.

Let $Z$ be the set of trees that can be constructed from the run of the algorithm using the table $T$. We prove the claim in the following two steps:

1. All trees in $Z$ are consistent with the table $T$.

2. If a number got recolored more than $t$ times then there exists a tree $\tau \in Y \cap Z$ which leads to the desired contradiction.

\section{All trees in $Z$ are consistent with the table $T$ :}

We want to argue that the colors that gets filled from $T$ into a $k$-AP $E$ when consistency is checked are exactly the same entries in $T$ that the algorithm sees before it recolors this $k$-AP $E$, i.e., both are monochromatic. Focusing on one number $x \in E$ we directly see that the entry from $T$ that is used to recolor $x$ is the entry with the number $i$ from the column in $T$ that belongs to $x$, where $i$ is the number of times a color for $x$ was needed before which is exactly one plus the number of $k$-APs containing $x$ that got recolored before. Note that all these $k$-APs appear in a tree below $E$ which is the reason why when consistency is checked for also exactly the entry $i$ is filled into $x$ (see definition of consistency). This proves that any tree that got created from a run with table $T$ is consistent with $T$.

If a number got recolored more than $t$ times then a tree $\tau \in Y$ is constructed: 
For sake of contradiction we assume that a number got recolored more than $t$ times and argue that in this case a tree $\tau \in Y$ gets constructed. Note that by construction all trees fulfill the properties 1-4. Hence it remains that a tree of size between $t$ and $2 t$ is generated from the trace. For this let $\tau$ be the the smallest tree in $Z$ of size $s \geq t$. Such a tree exists because generating a tree from the $t^{\text {th }}$ time a number got recolored produces a tree of size at least $t$. If the label $S$ of the root of $\tau$ consists of just one number then because of property 3 and 4 it has only one child and the tree generated choosing this child as a root label has size $s-1$. Otherwise take one number $x \in S$ and look at the trees generated with $\{x\}$ and $S-\{x\}$ as a root label. One of them has size at least $s / 2$ since each node in the tree generated by $S$ appears in at least one of the new trees. In either case the minimality of $\tau$ - that the remaining tree of size either $s-1$ or $s / 2$ has to be smaller than $t$-implies $s \leq 2 t$. This shows that the tree $\tau$ that is constructed from the trace fulfills all 5 properties and is therefore a tree from $Y$ that is consistent with the table $T$. This is a contradiction to the way we constructed the table $T$ in stage 2 .

\section{End of Proof of Claim}

It is easy to see that with the guarantee given by this claim the algorithm runs for at most $O(t n)$ time in this stage and terminates with a proper coloring. With all previous stages running in time polynomial in $n$ the entire algorithm does so and thus fulfills the properties of a deterministic-constructive proof, finishing the proof of Theorem 7.1.

Corollary 7.2 $W(k, 2) \geq \frac{2^{(k-1)}}{e k}-1$ by a randomized-constructive proof.

Proof: The algorithm used to achieve this bound is simply stage 3 of the algorithm above but instead of using the colors from a carefully prepared table $T$ an independent uniformly random color is chosen each time a new color is needed. If more than $t$ new colors are requested for any number the algorithm stops and reports its failure. This is the randomized algorithm of Moser and Tardos [18] which is very similar and actually encompasses Moser's algorithm given in Section 6. For its analysis we note that the only reason why Theorem 7.1 does not give us the bound of this theorem is because we can not make $\epsilon$ smaller with $k$. The reason for this is that the running time of stage 1 and 2 is $n^{O\left(\epsilon^{-1.01}\right)}$ time which forces $\epsilon$ to be a fixed constant. Since the randomized algorithm only runs stage 3 we can choose $\epsilon=\Theta\left(\frac{1}{n^{\prime} k}\right)$ where $n^{\prime}=\frac{2^{(k-1)}}{e k}$ such that $2^{-(k-1) \epsilon} \geq e^{-n^{\prime}} \geq$ $\left(1-1 / n^{\prime}\right)$. This still keeps the running time of stage 3 to be polynomial, more specifically $O(t n)=O\left(n \epsilon^{1.01}\right)=O\left(n\left(n^{\prime} k\right)^{1.01}\right)$. The success probability comes directly from the analysis for stage 2 . There is already stated that the probability for random colors to form a good table is at least $2 / 3$. Thus also the success probability of the described algorithm to reports a proper 2-coloring is as required by the definition of randomizedconstructive. With such a small $\epsilon$ the lower bound implied by this randomized algorithm now becomes $W(k, 2) \geq \frac{2^{(k-1)(1-\epsilon)}}{e k} \geq \frac{2^{(k-1)}}{e k}\left(1-1 / n^{\prime}\right)=\frac{2^{(k-1)}}{e k}-1$ as desired. 


\section{Acknowledgements}

We would like to thank Robin Moser for his brilliant talk at STOC 2009 which inspired this paper. We would also like to thank Thomas Dubois, Mohammad Hajiaghayi and Larry Washington for proofreading and helpful comments. Last but not least we would like to thank the anonymous reviewer(s) for catching several minor mistakes and for helpful suggestions which greatly improved the presentation of this paper.

\section{References}

[1] R. C. Baker, G. Harman, and J. Pintz. The difference between consecutive primes. II. Proc. London Math. Soc. (3), 83(3):532-562, 2001.

[2] V. Bergelson and A. Leibman. Polynomial extensions of van der Waerden's and Szemerédi's theorems. Journal of the American Mathematical Society, pages 725$753,1996$.

[3] E. Berlekamp. A construction for partitions which avoids long arithmetic progressions. CMB, 11:409-414, 1968.

[4] K. Chandrasekaran, N. Goyal, and B. Haeupler. Deterministic Algorithms for the Lovász Local Lemma. In Proceedings of the 21st ACM-SIAM Symposium on Discrete Algorithms (SODA '10), 2010.

[5] P. Erdös and L. Lovász. Problems and results on 3-chromatic hypergraphs and some related questions. Infinite and finite sets, 2:609-627, 1975.

[6] P. Erdös and R. Rado. Combinatorial theorems on classifications of subsets of a given set. In Proceedings of the London Mathematical Society, 2:417-439, 1952.

[7] P. Erdös and J. Selfridge. On a combinatorial game. Journal of Combinatorial Theory, Series A, 14(3):298-301, 1973.

[8] W. Gasarch, C. Kruskal, and A. Parrish. Purely combinatorial proofs of van der Waerden-type theorems. www.gasarch.edu/ gasarch/ vdw/vdw.html.

[9] W. Gowers. A new proof of Szemerédi's theorem. Geometric and Functional Analysis, 11:465-588, 2001.

[10] R. Graham, B. Rothchild, and J. Spencer. Ramsey Theory. Wiley, 1990.

[11] B. Haeupler, B. Saha and A. Srinivasan. New Constructive Aspects of the Lovász Local Lemma. In Proceedings of the 51st IEEE Symposium on Foundations of Computer Science (FOCS '10), 2010.

[12] D. R. Heath-Brown. Differences between consecutive primes. Jahresber. Deutsch. Math.-Verein., 90(2):71-89, 1988.

[13] R. Impagliazzo and A. Wigderson. Randomness vs time: derandomization under a uniform assumption. Journal of Computer and System Sciences, 65:672-694, 2002.

[14] D. Knuth. The Art of Computer Programming, Volume 1: Fundamental Algorithms. Addison-Wesley, Reading, MA, 1969. 
[15] Li and Vitányi. An introduction to Kolmogorov complexity and its applications (3rd edition). Springer, New York, 2008.

[16] R. Moser. A constructive proof of the general Lovász Local Lemma, 2009. Slides for the talk at STOC 2009, which differ from the paper.

[17] R. Moser. A constructive proof of the Lovász Local Lemma. In Proceedings of the 41st ACM Symposium on Theory of Computing (STOC'09), pages 343-350, 2009.

[18] R. Moser and G. Tardos. A constructive proof of the general Lovász Local Lemma. Journal of the ACM, 57(2):1-15, 2010.

[19] N. Nisan and A. Wigderson. Hardness vs randomness. Journal of Computer and System Sciences, 49, 1994.

[20] R. Rado. Studien zur Kombinatorik. Mathematische Zeitschrift, pages 424-480, 1933.

[21] R. Rado. Notes on Combinatorial Analysis. In Proceedings of the London Mathematical Society, pages 122-160, 1943.

[22] P. Schweitzer. Using the incompressibility method to obtain local lemma results for Ramsey-type problems. Information Processing Letters, 109, 2009.

[23] S. Shelah. Primitive recursive bounds for van der Waerden numbers. Journal of the American Mathematical Society, pages 683-697, 1988.

[24] Z. Szabó. An application of Lovász Local Lemma - a new lower bound on the van der Waerden numbers. Random Structures and Algorithms, 1, 1990.

[25] B. van der Waerden. Beweis einer Baudetschen Vermutung. Nieuw Arch. Wisk., 15:212-216, 1927.

[26] M. Walters. Combinatorial proofs of the polynomial van der Waerden theorem and the polynomial Hales-Jewett theorem. Journal of the London Mathematical Society, $61: 1-12,2000$. 